\title{
Spreading of Extended-Spectrum Beta-Lactamases Among the Klebsiella pneumoniae Strains Isolated from Outpatients With Urinary Tract Infection in Zahedan, Southeast Iran
}

\author{
Shahram Shahraki-Zahedani, ${ }^{1}$ Mehdi Moghadampour, ${ }^{2,}$ Mohammad Bokaeian, ${ }^{3}$ and Alireza \\ Ansari-Moghaddam ${ }^{4}$ \\ ${ }^{1}$ Infectious Diseases and Tropical Medicine Research Center, Zahedan University of Medical Sciences, Zahedan, IR Iran \\ ${ }^{2}$ Department of Microbiology, School of Medicine, Zahedan University of Medical Sciences, Zahedan, IR Iran \\ ${ }^{3}$ Department of Laboratory Sciences, School of Paramedical Sciences, Zahedan University of Medical Sciences, Zahedan, IR Iran \\ ${ }^{4}$ Health Promotion Research Center, Zahedan University of Medical Sciences, Zahedan, IR Iran \\ "Corresponding author: Mehdi Moghadampour, Department of Microbiology, School of Medicine, Zahedan University of Medical Sciences, Zahedan, IR Iran. E-mail: \\ mehdimoghadampoor@yahoo.com
}

Received 2015 May 14; Accepted 2015 September 5.

\begin{abstract}
Background: Extended-spectrum beta-lactamases (ESBLs) represents a significant and rapid emerging problem around the world. Nowadays, antibiotic resistance determinants are prevalent in both hospital and community strains of Gram-negative enteric bacteria.

Materials and Methods: In this sectional-descriptive study, 87 clinical isolates of Klebsiella pneumoniae were collected from outpatients and their susceptibility to antimicrobial agents, minimum inhibitory concentration (MIC) values for third-generation cephalosporins and ESBLs production were assessed.

Results: Of 87 K. pneumoniae clinical isolates, 20 (22.9\%) isolates represented the ESBLs phenotype.

Conclusions: Our results demonstrated that the resistance to the third-generation cephalosporins in K. pneumoniae strains isolated from outpatients is relatively high in Zahedan.
\end{abstract}

Keywords: Extended-Spectrum Beta-Lactamases, Urinary Tract Infection, Klebsiella pneumoniae

\section{Background}

KLebsiella pneumoniae is a member of the Enterobacteriaceae family. K. pneumoniae is ubiquitous in nature and can be found in natural environments and on mucosal surfaces of mammals. This microorganism is an opportunistic bacterial pathogen that results in urinary tract infections in humans commonly [1,2]. Antibiotic resistance is a growing concern that threatens the effective treatment of infectious diseases. Extended-spectrum beta-lactamases (ESBLs) are bacterial plasmid encoded enzymes that cause bacterial resistance against the penicillins, the first, second and third generation cephalosporins, as well as aztreonam by hydrolyzing these antibiotics. ESBLs are inhibited by beta-lactamase inhibitors such as clavulanic acid [3]. ESBL-producing isolates are commonly resistant to multiple antibiotics used for treating infections resulted from Gram-negative bacteria [4].

Spreading of ESBLs among clinical isolates of Enterobacteriaceae and especially $K$. pneumoniae strains has become a worldwide problem [5]. ESBLs have been extensively identified as a vital threat among multidrugresistant bacterial isolates [6]. Nowadays, ESBLs are preva- lent in K. pneumoniae strains isolated from both inpatients and outpatients. Several studies have been conducted in Iran to determine the prevalence of ESBLs in hospital strains of K. pneumoniae [7-11].

\section{Objectives}

The present investigation was carried out to study the prevalence of ESBLs among K. pneumoniae strains isolated from outpatients with urinary tract infection by phenotypic tests.

\section{Materials and Methods}

In this sectional-descriptive study, 87 unrepeated clinical strains of K. pneumoniae were isolated from collected urine samples of young women with urinary tract infection. All patients were referred to microbiology laboratories of three major hospitals (Ali ibn Abi Talib, Khatam al Anbiya and Buali hospitals) of Zahedan during November 2011 to November 2012. All patients were interviewed with 
regard to their urogenital symptoms. None had any history of hospitalization. The bacterial isolates were recognized using usual biochemical tests [2]. Standard bacterial strains including K. pneumoniae ATCC 700603 as positive control as well as Escherichia coli ATCC 25922 and Pseudomonas aeruginosa ATCC 27853 as negative control in antibiotic susceptibility testing were prepared from american type culture collection center.

To determine the antimicrobial susceptibility of isolates, all isolates were plated on Mueller-Hinton agar and their susceptibilities to 16 different antibiotics were tested by Kirby-Bauer disk diffusion assay and the results were interpreted according to the clinical and laboratory standards institute (CLSI) procedure [12]. The used disks (MAST, Merseyside, UK) were included cefotaxime (30 $\mu \mathrm{g})$, ceftazidime (30 $\mu \mathrm{g})$, ceftriaxone (30 $\mu \mathrm{g}$ ), cefpodoxime (10 $\mu \mathrm{g})$, cefepime (30 $\mu \mathrm{g})$, aztreonam (30 $\mu \mathrm{g})$, imipenem (10 $\mu \mathrm{g})$, gentamicin $(10 \mu \mathrm{g})$, streptomycin $(10 \mu \mathrm{g})$, amikacin (30 $\mu \mathrm{g})$, ciprofloxacin $(5 \mu \mathrm{g})$, nalidixic acid (30 $\mu \mathrm{g})$, nitrofurantoin $(300 \mu \mathrm{g})$, colistin sulfate $(25 \mu \mathrm{g})$, trimethoprim/sulfamethoxazole (1.25/23.75 $\mu \mathrm{g}$ ) and amoxicillin (25 $\mu \mathrm{g})$.

For screening ESBL-producing isolates, the CLSI confirmatory test was done by disk diffusion method with disks containing ceftazidime ( $30 \mu \mathrm{g})$ and ceftazidime/clavulanic acid $(30 / 10 \mu \mathrm{g})$, as well as cefotaxime (30 $\mu \mathrm{g})$ and cefotaxime/clavulanic acid (30/10 $\mu \mathrm{g})[12]$.

Minimum inhibitory concentration (MIC)-values of four antibiotics consist of cefotaxime, ceftazidime, ceftriaxone and cefpodoxime were determined for isolates with ESBLs positive phenotype by the E-test method (Liofilchem MIC test strips, Italy). Concentration gradient for cefotaxime was $0.002-32 \mu \mathrm{g} / \mathrm{mL}$, and for ceftazidime, ceftriaxone and cefodoxime were $0.016-256 \mu \mathrm{g} / \mathrm{mL}$. Interpretive criteria were as per the E-test manufacturer's guidelines and CLSI. E. coli ATCC 25922 was used as quality control for MIC test strips [13].

All descriptive statistical analyses were performed by applying the SPSS-18 and Excel 2013 software.

\section{Results}

The pattern of antibiotic resistance of all isolates for 16 different antibiotic agents was demonstrated in Table 1.

In initial disk diffusion test of isolates for cefotaxime, ceftazidime, ceftriaxone, cefpodoxime and aztreonam, 23 (26.4\%) isolates have shown reduced sensitivity among which 20 (22.9\%) isolates were passed the CLSI confirmatory test (combination disk), thus recognized as ESBLsproducers. The disk diffusion assay for ESBL-producer isolates revealed that all isolates had multidrug resistance.
Characteristics of these isolates as well as their MIC-values for utilized antibiotics were demonstrated in Table 2.

\section{Discussion}

K. pneumoniae is one of the major ESBLs-producing bacteria. The highest prevalence of ESBLs-producing K. pneumoniae is seen in Asian countries including Iran. Our results showed that $22.9 \%$ of K. pneumoniae strains isolated from outpatients produced the ESBLs. In our previous study, the prevalence of ESBLs among K. pneumoniae strains isolated from inpatients was 40.78\% [10].

In comparison to prior results, the prevalence of ESBLs among strains isolated from outpatients was almost half of the frequency of these enzymes in the strains isolated from inpatients; hence, this is a great risk to this region.

All isolates were resistant to amoxicillin. No isolates were resistant to imipenem and colistin sulfate. In addition, among the tested strains, the lowest antibiotic resistance (under $10 \%$ ) was observed to amikacin, gentamicin and ciprofloxacin, respectively. Therefore, regarding to the side effects of imipenem and colistin sulfate, it can be said these antibiotics (aminoglycosides and fluoroquinolones) are the best therapeutic options for treatment of outpatients with urinary tract infection in Zahedan.

Our finding suggests that ESBLs-producing bacteria have found their way out of the hospital and this is a great danger to the society. Epidemiological studies propose that the progressive extensive use of third-generation cephalosporins is a major risk factor that contributed to the emergence of ESBLs-producing K. pneumoniae [1]. Antibiotic overuse in humans and animals, hospital crossinfection, the food chain, trade and human migration appears to play a role in recent spreading of ESBLs outside hospitals, although the role of these factors is unclear and is related to specific epidemiological situations [14]. Our study shows that of 16 antibiotics tested, the ESBLsproducing strains were resistant to the minimum andmaximumnumberof 6 and 12 antibiotics, respectively. These results propose the transfer of multi-drug resistance plasmids among Gram-negative bacteria [1], thus endangering the treatment of infections caused by these strains. In conclusion, our results indicate that the ESBLs have been developed in both bacterial strains isolated from inpatients or outpatients in this region and health care system should have more control over the use of these antibiotics. In this study, we did not perform genetic experiments for epidemiology purposes, and this is the limitation of our study. 
Table 1. Antimicrobial Susceptibilities of the K. pneumoniae Isolates

\begin{tabular}{|c|c|c|c|c|}
\hline Antibiotic & Sensitive, No. (\%) & Intermediate, No. (\%) & Resistant, No. (\%) & Total, No. (\%) \\
\hline Cefotaxime & $66(75.9)$ & $1(1.1)$ & $20(23.0)$ & $87(100)$ \\
\hline Ceftazidime & $68(78.2)$ & $2(2.3)$ & $17(19.5)$ & $87(100)$ \\
\hline Ceftriaxone & $66(75.9)$ & $1(1.1)$ & $20(23.0)$ & $87(100)$ \\
\hline Cefpodoxime & $66(75.9)$ & $0(0.0)$ & $21(24.1)$ & $87(100)$ \\
\hline Cefepime & $74(85.1)$ & $6(6.9)$ & $7(8.0)$ & $87(100)$ \\
\hline Aztreonam & $65(74.7)$ & $2(2.3)$ & $20(23.0)$ & $87(100)$ \\
\hline Imipenem & $87(100.0)$ & $0(0.0)$ & $0(0.0)$ & $87(100)$ \\
\hline Amoxicillin & $0(0.0)$ & $0(0.0)$ & $87(100.0)$ & $87(100)$ \\
\hline Nalidixic acid & $72(82.8)$ & $6(6.9)$ & $9(10.3)$ & $87(100)$ \\
\hline Ciprofloxacin & $79(90.8)$ & $2(2.3)$ & $6(6.9)$ & $87(100)$ \\
\hline Nitrofurantoin & $36(41.4)$ & $4(4.6)$ & $47(54.0)$ & $87(100)$ \\
\hline Trimethoprim/sulfamethoxazole & $60(69.0)$ & $1(1.1)$ & $26(29.9)$ & $87(100)$ \\
\hline Gentamicin & $81(93.1)$ & $0(0.0)$ & $6(6.9)$ & $87(100)$ \\
\hline Streptomycin & $72(82.8)$ & $3(3.4)$ & $12(13.8)$ & $87(100)$ \\
\hline Amikacin & $86(98.9)$ & $0(0.0)$ & $1(1.1)$ & $87(100)$ \\
\hline Colistin sulfate & $87(100.0)$ & $0(0.0)$ & $0(0.0)$ & $87(100)$ \\
\hline
\end{tabular}

Table 2. Characteristics of ESBLs-Producing K. pneumoniae Isolates $(\mathrm{n}=20)$

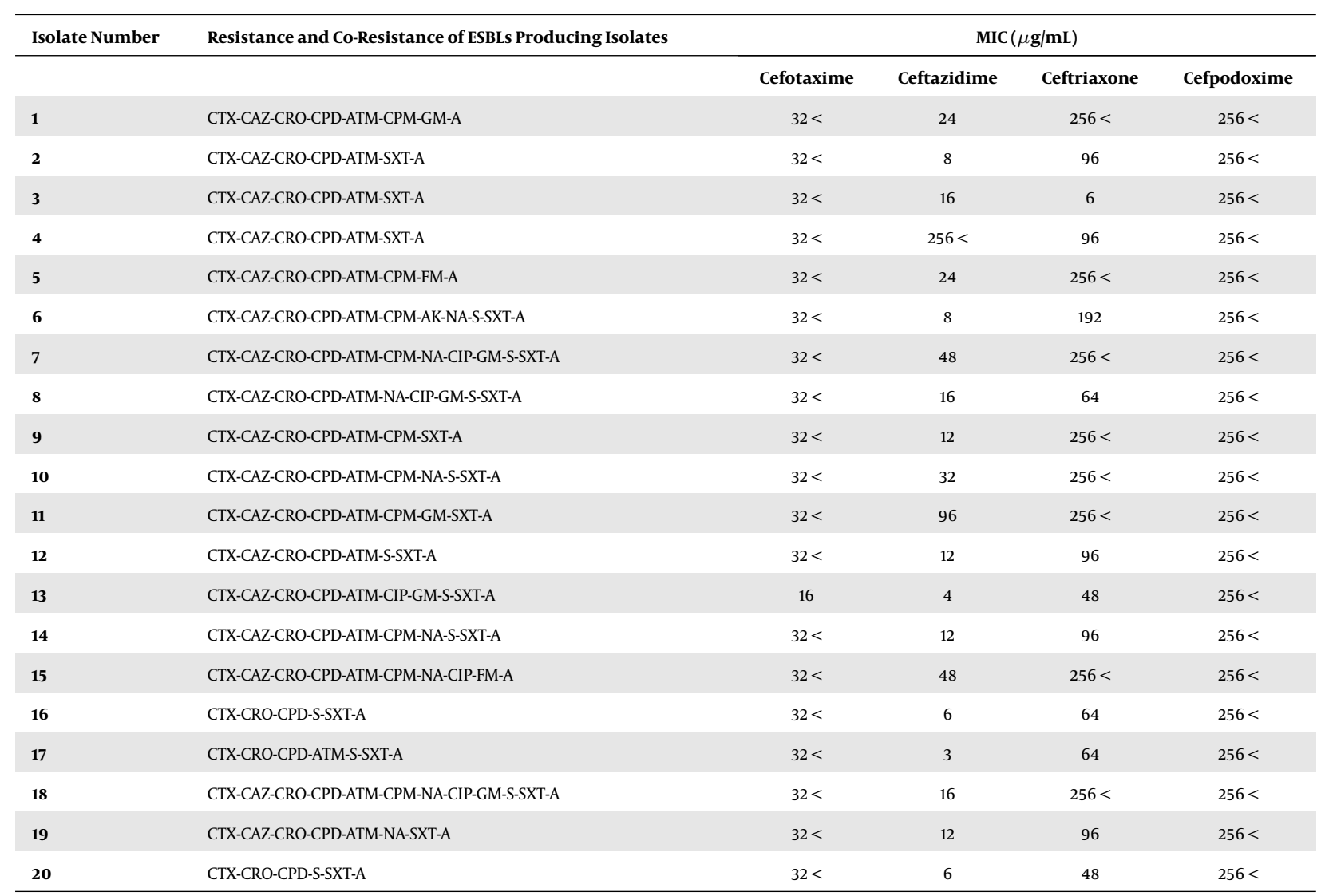

Abbreviations: AK: amikacin, A: amoxicillin, ATM: aztreonam, IMI: imipenem, CAZ: ceftazidime„ CIP: ciprofloxacin, CRO: ceftriaxone, CPM: cefepime, CPD: cefpodoxime, CTX: cefotaxime, FM: nitrofurantoin, GM: gentamicin, NA: nalidixic acid, S: streptomycin, SXT: trimethoprim/sulfamethoxazole. 


\section{Acknowledgments}

The authors appreciate all individuals who willingly participated in the current study. This article is the result of research project No. 2498 (Executor of project: Dr. Shahram Shahraki-Zahedani).

\section{Footnotes}

Authors' Contribution: Shahram Shahraki-Zahedani managed the research. Mehdi Moghadampour performed the tests and wrote the paper. Mohammad Bokaeian helped in managing the research. Alireza AnsariMoghaddam contributed to data analysis.

Funding/Support: Zahedan University of Medical Sciences, Zahedan, IR Iran.

\section{References}

1. Gupta A, Ampofo K, Rubenstein D, Saiman L. Extended spectrum beta lactamase-producing Klebsiella pneumoniae infections: a review of the literature.J Perinatol. 2003;23(6):439-43. doi:10.1038/sj.jp.7210973. [PubMed: 13679928].

2. Podschun R, Ullmann U. Klebsiella spp. as nosocomial pathogens: epidemiology, taxonomy, typing methods, and pathogenicity factors. Clin Microbiol Rev. 1998;11(4):589-603. [PubMed: 9767057].

3. Paterson DL, Bonomo RA. Extended-spectrum beta-lactamases: a clinical update. Clin Microbiol Rev. 2005;18(4):657-86. doi: 10.1128/CMR.18.4.657-686.2005. [PubMed:16223952].

4. Qi C, Pilla V, Yu JH, Reed K. Changing prevalence of Escherichia coli with CTX-M-type extended-spectrum beta-lactamases in outpatient urinary E. coli between 2003 and 2008. Diagn Microbiol Infect Dis. 2010;67(1):87-91. doi: 10.1016/j.diagmicrobio.2009.12.011. [PubMed: 20227224].

5. Bradford PA. Extended-spectrum beta-lactamases in the 21st century: characterization, epidemiology, and detection of this important resistance threat. Clin Microbiol Rev. 2001;14(4):933-51. doi: 10.1128/CMR.14.4.933-951.2001. [PubMed: 11585791] table of contents.
6. Canton R, Novais A, Valverde A, Machado E, Peixe L, Baquero F, et al. Prevalence and spread of extended-spectrum beta-lactamaseproducing Enterobacteriaceae in Europe. Clin Microbiol Infect. 2008;14 Suppl 1:144-53. doi: 10.1111/j.1469-0691.2007.01850.x. [PubMed: 18154538].

7. Nematzadeh S, Shahcheraghi F, Feizabadi MM, Nikbin VS, Nasehi L. Molecular characterization of CTX-Mbeta-lactamases among Klebsiella pneumoniae isolated from patients at Tehran hospitals. Indian J Med Microbiol. 2011;29(3):254-7. doi: 10.4103/0255-0857.83908. [PubMed: 21860105].

8. Feizabadi MM, Delfani S, Raji N, Majnooni A, Aligholi M, Shahcheraghi F, et al. Distribution of bla(TEM), bla(SHV), bla(CTX-M) genes among clinical isolates of Klebsiella pneumoniae at Labbafinejad Hospital, Tehran, Iran. Microb Drug Resist. 2010;16(1):49-53. doi: 10.1089/mdr.2009.0096. [PubMed:19961397].

9. Ghafourian S, Sekawi Z, Neela V, Khosravi A, Rahbar M, Sadeghifard $\mathrm{N}$. Incidence of extended-spectrum beta-lactamase-producing Klebsiella pneumoniae in patients with urinary tract infection. Sao Paulo Med J. 2012;130(1):37-43. [PubMed: 22344358].

10. Shahraki-Zahedani S, Moghadampour M, Bokaeian M, Ansari-Moghaddam A. Prevalence of CTX-M-8 and CTX-M15 type extended-spectrum beta-lactamases between Klebsiella pneumoniae spp. isolated from Zahedan, Southeast Iran. J Chemother. 2015:1973947815Y0000000008. doi: 10.1179/1973947815Y.0000000008. [PubMed: 25801185].

11. Bazzaz BS, Naderinasab M, Mohamadpoor AH, Farshadzadeh Z, Ahmadi S, Yousefi F. The prevalence of extended-spectrum betalactamase-producing Escherichia coli and Klebsiella pneumoniae among clinical isolates from a general hospital in Iran. Acta Microbiol Immunol Hung. 2009;56(1):89-99. doi: 10.1556/AMicr.56.2009.1.7. [PubMed: 19388560].

12. Cockerillm FR, Wiklerm MA, Alder J. Performance Standards for Antimicrobial Disk Susceptibility Tests. 11 ed. Wayne: Clinical and Laboratory Standards Institute; 2012. pp. 1-76.

13. Manoharan A, Premalatha K, Chatterjee S, Mathai D, Sari Study Group . Correlation of TEM, SHV and CTX-M extended-spectrum beta lactamases among Enterobacteriaceae with their in vitro antimicrobial susceptibility. Indian J Med Microbiol. 2011;29(2):161-4. doi: 10.4103/0255-0857.81799. [PubMed: 21654112].

14. Coque TM, Baquero F, Canton R. Increasing prevalence of ESBLproducing Enterobacteriaceae in Europe. Euro Surveill. 2008;13(47) [PubMed: 19021958]. 